Research

Open Access

\title{
Quality of life before intensive care unit admission is a predictor of survival
}

\author{
José GM Hofhuis ${ }^{1,2}$, Peter E Spronk ${ }^{1}$, Henk F van Stel ${ }^{3,4}$, Augustinus JP Schrijvers ${ }^{3}$ and \\ Jan Bakker²
}

\begin{abstract}
1Department of Intensive Care Medicine, Gelre Hospitals (location Lukas), Albert Schweitzerlaan, 7334 DZ Apeldoorn, The Netherlands 2Department of Intensive Care Medicine, Erasmus Medical Centre, Gravendijkwal 230, Rotterdam, 3015 CE, The Netherlands

3Julius Center for Health Sciences and Primary Care, University Medical Centre Utrecht, Heidelberglaan 100, Utrecht, 3584 CX, The Netherlands

${ }^{4}$ Department of Medical Decision Making, Leiden University Medical Centre, Albinusdreef 2, Leiden, 2333 ZA, The Netherlands
\end{abstract}

Corresponding author: José GM Hofhuis, j.hofhuis@gelre.nl

Received: 5 Mar 2007 Revisions requested: 5 Apr 2007 Revisions received: 22 Jun 2007 Accepted: 13 Jul 2007 Published: 13 Jul 2007

Critical Care 2007, 11:R78 (doi:10.1186/cc5970)

This article is online at: http://ccforum.com/content/11/4/R78

(C) 2007 Hofhuis et al:; licensee BioMed Central Ltd.

This is an open access article distributed under the terms of the Creative Commons Attribution License (http://creativecommons.org/licenses/by/2.0), which permits unrestricted use, distribution, and reproduction in any medium, provided the original work is properly cited.

\begin{abstract}
Introduction Predicting whether a critically ill patient will survive intensive care treatment remains difficult. The advantages of a validated strategy to identify those patients who will not benefit from intensive care unit (ICU) treatment are evident. Providing critical care treatment to patients who will ultimately die in the ICU is accompanied by an enormous emotional and physical burden for both patients and their relatives. The purpose of the present study was to examine whether health-related quality of life (HRQOL) before admission to the ICU can be used as a predictor of mortality.

Methods We conducted a prospective cohort study in a university-affiliated teaching hospital. Patients admitted to the ICU for longer than 48 hours were included. Close relatives completed the Short-form 36 (SF-36) within the first 48 hours of admission to assess pre-admission HRQOL of the patient. Mortality was evaluated from ICU admittance until 6 months after ICU discharge. Logistic regression and receiver operating characteristic analyses were used to assess the predictive value for mortality using five models: the first question of the SF-36 on general health (model A); HRQOL measured using the physical component score (PCS) and mental component score (MCS) of the SF-36 (model B); the Acute Physiology and Chronic Health Evaluation (APACHE) II score (an accepted mortality prediction model in ICU patients; model C); general health and APACHE II score (model D); and PCS, MCS and APACHE II score (model

E). Classification tables were used to assess the sensitivity, specificity, positive and negative predictive values, and likelihood ratios.

Results A total of 451 patients were included within 48 hours of admission to the ICU. At 6 months of follow up, 159 patients had died and 40 patients were lost to follow up. When the general health item was used as an estimate of HRQOL, area under the curve for model $A(0.719)$ was comparable to that of model $C(0.721)$ and slightly better than that of model $D$ (0.760). When PCS and MCS were used, the area under the curve for model B (0.736) was comparable to that of model $C$ (0.721) and slightly better than that of model $E$ (0.768). When using the general health item, the sensitivity and specificity in model $D$ (sensitivity 0.52 and specificity 0.81 ) were similar to those in model $A(0.45$ and 0.80$)$. Similar results were found when using the MCS and PCS.

Conclusion This study shows that the pre-admission HRQOL measured with either the one-item general health question or the complete SF-36 is as good at predicting survival/mortality in ICU patients as the APACHE II score. The value of these measures in clinical practice is limited, although it seems sensible to incorporate assessment of HRQOL into the many variables considered when deciding whether a patient should be admitted to the ICU.
\end{abstract}

\section{Introduction}

It is difficult for doctors to predict whether a critically ill patient will survive intensive care treatment. Mortality in patients admitted to intensive care units (ICU) remains high [1]. An increasing number of in-hospital patients die in the ICU [2]. The advantages of a validated strategy to identify those

APACHE = Acute Physiology and Chronic Health Evaluation; AUC = area under the curve; HRQOL = health-related quality of life; ICU = intensive care unit; LASA = linear analogue self assessment; MCS = mental component score; PCS = physical component score. 
patients who will not benefit from ICU treatment are evident. Providing critical care treatment to patients who will ultimately die in the ICU is accompanied by an enormous emotional and physical burden for both patients and their relatives. Furthermore, ICU resources are scarce, and identifying those patients who will not survive intensive care treatment allows us to make better use of what resources are available [3]. The available predicting tools, including the Acute Physiology and Chronic Health Evaluation (APACHE) II score, are based on a combination of pre-morbid factors and acute physiology items recorded during the first 24 hours after admission. The use of these systems in individual patients is limited because they have been validated at the group level. Consequently, ICU doctors must rely upon their clinical experience in their decision making. The predictive value of clinical experience in this regard is also limited [4]. We hypothesized that the perceived health-related quality of life (HRQOL) of patients also reflects components of 'physiological reserve' and could, as such, act as a predictor of mortality.

The goal of the present study was to evaluate the predictive value for survival of the pre-admission HROOL, alone and in combination with the APACHE II score, in critically ill patients.

\section{Materials and methods}

All patients admitted for more than 48 hours to the 10-bed mixed surgical-medical ICU of the Gelre Lukas hospital in Apeldoorn (a 654-bed, university-affiliated hospital in The Netherlands) were eligible for the study. We included only patients with a ICU stay of longer than 48 hours because we aimed to evaluate the sickest patients, hypothesizing that those patients were more likely to die. We felt that proxies of patients who would die during the first 48 hours after ICU admission should not be burdened with study participation. Between September 2000 and April 2004, all admitted patients were screened for eligibility for study participation (Figure 1). The local ethics committee approved the study. Informed consent was given by a close relative and as soon as possible by the patient. Mortality was evaluated from ICU admittance until 6 months after ICU discharge. The severity of illness was routinely measured using the APACHE II score [5]. Physicians treating the patients were not aware of the preadmission HRQOL.

\section{Health-related quality of life measurement}

The Short-form 36 (SF-36, version 1; ${ }^{\circ} 1993$ Medical Outcome Trust), a generic, widely used standardized health status questionnaire, was used to measure HRQOL. This measurement contains eight multi-item dimensions: physical functioning, role limitation due to physical problems, bodily pain, general health, vitality, social functioning, role limitation due to emotional problems, and mental health. Answers to the 36 items were transformed, weighed and subsequently scored according to predefined guidelines [6]. Higher scores represent better functioning, with a range from 0 to 100 . Fur-
Figure 1

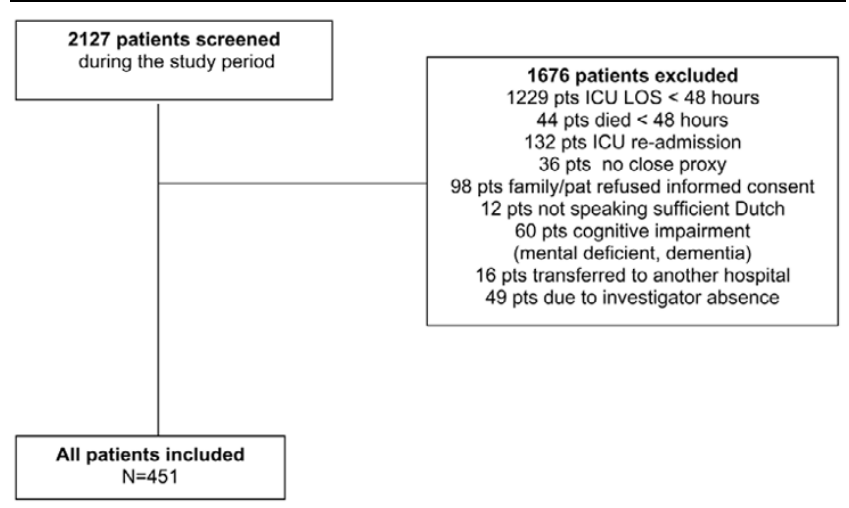

Flow diagram of patient selection and inclusion. Follow up was lost in 40 patients, usually because the patients did not live in the area of the hospital (they were on vacation). Characteristics of those patients did not differ from those of the group analyzed in the study (data not shown). A large group of patients $(n=1,229)$ were admitted to the intensive care unit (ICU) for under 48 hours and hence were excluded from the final analysis. Patients who died within 48 hours of ICU admission $(n=44)$ were excluded. In some cases the patient had no close proxy $(n=36)$. Patients re-admitted to the ICU were excluded ( $n=$ 132) because it was possible that the first admission could have biased the proxy memories of the patient's pre-admission health-related quality of life (HRQOL). Proxies or the patients themselves refused informed consent $(n=98)$ mainly because they felt study participation to be too great a burden at that stressful moment. Patients transferred to other hospitals $(n=16)$ or with cognitive impairment $(n=60)$, or who did not speak sufficient Dutch $(n=12)$ were also excluded. Some patients were not included because of investigator absence $(n=49)$. LOS, length of stay.

thermore, scores were aggregated to summary measures representing a physical component score (PCS; mainly reflecting physical functioning) and a mental component score (MCS; mainly reflecting social functioning and mental health) [7]. Population scores on PCS and MCS have been standardized on 50 as population mean (SD 10 representing 1) [7]. For the PCS, very high scores indicate no physical limitations, disabilities, or decrements in well being, as well as high energy levels. Very low scores indicate substantial limitations in self-care and in physical, social and role activities, severe bodily pain, or frequent tiredness [7]. For the MCS, very high scores indicate frequent positive effect, absence of psychological distress, and limitations in usual social/role activities caused by emotional problems. Very low scores indicate frequent psychological distress, and substantial social and role disability due to emotional problems [7].

Translation, validation and generating normative data of the Dutch language version of the SF-36 health questionnaire were evaluated in 1998 in community and chronic disease populations [8]. Because most of the patients in our study were unable to complete a questionnaire at the time of admission, proxies had to be used as a surrogate approach. In proxies and patients the same method was used to complete the SF-36. The use of proxies to assess the patients' HRQOL 
using the SF-36 in the ICU setting was validated in earlier studies conducted by our group [9] and others $[10,11]$. HRQOL was measured within 48 hours of ICU admission (estimation of HRQOL up to 4 weeks before admission). All interviews were performed by the same investigator $(\mathrm{JH})$. The average time required to complete the questionnaire was 15 to $20 \mathrm{~min}$. Consideration of multiple items has the advantage of allowing construction of a comprehensive profile of HRQOL, but it may burden the critically ill patient. We used the first question of the SF-36 as a primary approach to estimation of the patient's HRQOL. This is the single-item question pertaining general health status; 'In general, would you say your health is excellent, very good, good, fair, or poor?' $[12,13]$. The advantages of such a single-item question are its simplicity and ease of application.

\section{Statistical analysis}

A Pearson's $\chi^{2}$ test was used to assess demographic differences between ICU survivors and ICU non survivors. The differences between scores for the single-item question were tested using the $\chi^{2}$ test for trend. We examined the relationship between the single-item question on HROOL before ICU admission and mortality at 6 months after ICU discharge with multivariate logistic regression using the variables known on the first day of ICU admission (APACHE II score), adjusted for age and sex.

To analyze the potential of variables to predict mortality in patient subgroups, we used five statistical models. HRQOL was entered as the response to the single-item question, or as MCS and PCS. In the model $A$ we included the general health item of the SF-36, age and sex. In model B we included both the PCS and MCS from the SF-36, and age and sex. In model $C$ we included APACHE II score, age and sex. In model D we included the general health item of the SF-36, APACHE II score, age and sex. In model E we included both the PCS and MCS from the SF-36, APACHE-II score, age and sex.

To estimate the ability to discriminate between survivors and non-survivors, odds ratios were calculated, receiver operating characteristic analysis was performed and the area under the curve (AUC) was calculated. Classification tables were used to assess the sensitivity for observed deaths being labeled by the models as predicted deaths, specificity for a predicted death being an observed death, and positive and negative predictive values and likelihood ratio. Data were analyzed using SPSS (version 11.5; SPSS Inc., Chicago, IL, USA). All data are expressed as median (interquartile range), unless indicated otherwise.

$P<0.05$ was considered statistically significant.

\section{Results}

During the study period, 451 patients (61.2\% male and $38.8 \%$ female) were included. At 6 months after ICU discharge, 159
Table 1

Demographic and clinical characteristics

\begin{tabular}{|c|c|}
\hline Characteristic & Included patients $(n=451)$ \\
\hline Age (years)a & $71.0(63$ to 71$)$ \\
\hline Sex (male/female; \%) & $61.2 / 38.8$ \\
\hline APACHE II score ${ }^{a}$ & 19.0 (15 to 23$)$ \\
\hline ICU length of stay (days)a & $8.0(5$ to 16$)$ \\
\hline Hospital length of stay (days)a & $23.0(14$ to 40$)$ \\
\hline Ventilation days+ & 6.0 (3 to 13$)$ \\
\hline \multicolumn{2}{|l|}{ Type of admission (\%) } \\
\hline Nonsurgicalb & 53.2 \\
\hline Elective surgery ${ }^{c}$ & 8.7 \\
\hline Acute surgery ${ }^{d}$ & 38.1 \\
\hline
\end{tabular}

patients had died. Forty patients were lost to follow up (Figure 1). Demographic and clinical characteristics are shown in Table 1.

Of the 451 included patients, in a small proportion of patients $(n=23)$ pre-admission HRQOL was derived from the patients themselves, whereas all other SF-36 scores were obtained from proxies.

\section{Prediction models}

Using the single-item question on $\mathrm{HROOL}$ as a potential predictor of survival, the AUC for model A (0.719) was comparable to that for the APACHE II score (model C; 0.721) and slightly better than that in model $D(A \cup C=0.760)$, in which both factors were combined (Table 2 and Figure 2). Comparable results were obtained when calculating odds ratios (Table 3) and with analysis using MCS and PCS in models B and E. The sensitivity and specificity in model $D$ (sensitivity 0.52 and specificity 0.81 ) were similar to those in model A ( 0.45 and 0.80). Similar results were found when using PCS and MCS. In ICU patients $(n=451)$, sensitivity improved from 0.44 (model C; APACHE II score only) to 0.56 (model E; APACHE II score, and PCS and MCS), respectively. Results for specificity were similar, improving from 0.84 (model C; APACHE II score only) to 0.82 (model E; APACHE II score, and PCS and MCS). Similar results were also found when using the general health item (models A and D; Table 2). The negative and positive predictive values and likelihood ratios are shown in Table 2.

The scores on the single-item question pertaining to general health status before ICU admission were higher in survivors than in the patients who died $(P<0.001)$, with respect to all, that is: excellent (3.6\% of survivors versus $1.9 \%$ of those who 
Table 2

Statistical characteristics of mortality prediction models in ICU patients

\begin{tabular}{|c|c|c|c|c|c|}
\hline Characteristic & Model A & Model B & Model C & Model D & Model E \\
\hline Sensitivity & 0.45 & 0.50 & 0.44 & 0.52 & 0.56 \\
\hline Specificity & 0.80 & 0.81 & 0.84 & 0.81 & 0.82 \\
\hline PPV & 0.58 & 0.62 & 0.63 & 0.63 & 0.66 \\
\hline NPV & 0.70 & 0.72 & 0.70 & 0.73 & 0.75 \\
\hline AUC & 0.719 & 0.736 & 0.721 & 0.760 & 0.768 \\
\hline $\mathrm{LR}+(95 \% \mathrm{Cl})$ & 2.24 (1.66 to 3.02$)$ & 2.59 (1.93 to 3.48$)$ & 2.71 (1.95 to 3.77$)$ & 2.69 (2.00 to 3.60$)$ & 3.07 ( 2.28 to 4.12$)$ \\
\hline LR - $(95 \% \mathrm{Cl})$ & 0.69 (0.59 to 0.80$)$ & 0.62 (0.52 to 0.73$)$ & $0.67(0.58-0.78)$ & $0.59(0.50$ to 0.71$)$ & $0.54(0.45$ to 0.65$)$ \\
\hline
\end{tabular}

Model A included the general health item of the 36-item Short-form (SF-36), age and sex. Model B included the physical component score (PCS), mental component score (MCS), age and sex. Model $\mathrm{C}$ included the Acute Physiology and Chronic Health Evaluation (APACHE) II score, age and sex. Model D included the general health item of the SF-36, APACHE II score, age and sex. Model E included PCS, MCS, APACHE II score, age and sex. AUC, area under the curve; Cl, confidence interval; HRQOL, health-related quality of life; ICU, intensive care unit; $L R$, likelihood ratio (+positive, -negative); NPV, negative predictive value; PPV, positive predictive value.

died), very good $(5.6 \%$ versus $4.4 \%)$, good $(41.3 \%$ versus $18.9 \%)$, fair (38.1\% versus $50.9 \%)$, or poor (11.5\% versus $23.9 \%$ ). Other possibly relevant variables such as the presence of severe sepsis, length of ICU and hospital stay, and ventilation days were included in the logistic regression analysis. However, because these variables did not contribute significantly to the prediction models, they were omitted from the final models, as described above.

\section{Discussion}

We demonstrated that HRQOL before ICU admission can be used as a predictor of mortality in patients admitted to the ICU for longer than 48 hours. The mortality prediction ability of the pre-admission HRQOL estimated from the single-item question on the SF-36 was equal to those of the SF-36 (PCS and MCS) and the APACHE II score. Incorporating HRQOL into prediction models does not improve the predictive capacity of established models such as APACHE II and is not useful in clinical practice for making decisions in individual cases.

Mortality is difficult to predict for an individual patient because many factors determine survival from critical illness, such as age, sex, acute physiological deterioration and underlying illnesses. Several scoring systems aimed at predicting mortality have been developed that incorporate these factors. The APACHE II and III scores [5,14]., the Mortality Probability Model [15] and the Simplified Acute Physiology Score II [16] are established examples. When these systems were compared [17] their predictive ability, as judged by the AUC of the receiver operating characteristic curve, was around $70 \%$, which is comparable to our findings. However, these scoring systems are only available after 24 hours of ICU admission, and they are highly specific (able to predict survival [specificity $90 \%]$ ) but not very sensitive (less accurate in predicting death [sensitivity $50 \%$ to $70 \%$ ]) [4].
The advantages of using pre-admission HRQOL as a predictor of mortality are that it is easily obtained and available as soon as the patient, or a proxy (close family member), in the case of incapacity, can be questioned. In particular, a single item like the first question of the SF-36 is advantageous because of its simplicity and ease of administration in seriously ill patients. However, this benefit may be obtained at the cost of detail in the information provided. Multiple-item scoring systems such as the SF-36 have the advantage of providing a complete profile of HRQOL, although they are more laborious and carry the risk of asking potentially irrelevant questions [13]. These two types of items (multiple and single) could be used together in the clinical setting.

Can HRQOL be used as an indicator of final outcome? Several studies have addressed this question in dialysis patients [18-20], coronary artery bypass graft surgery patients [21], patients with congestive heart failure [22] and those with advanced colorectal cancer [23].

Currently, HRQOL surveys are rarely used in ICU clinical practice, and they predominantly address the impact that critical illness has on HRQOL after ICU survival. Only a few studies have focused on the association between pre-admission HRQOL and survival in critically ill patients [24-26]. Yinnon and coworkers [24] analyzed HRQOL in a 1-week period preceding ICU admission using the linear analogue self assessment (LASA) score. Mortality was higher in patients with lower LASA scores, indicating worse HRQOL, than in those with higher LASA scores, indicating a good HRQOL. However, the LASA was developed for application in cancer patients receiving chemotherapy, and it has not been validated for use in critically ill patients. In addition, the period of 1 week preceding ICU admission may be rather short to conduct an adequate evaluation of HRQOL pre-emptively. 
Table 3

Logistic regression models: odd ratios with $95 \%$ confidence intervals

\begin{tabular}{|c|c|c|c|}
\hline & OR & $95 \% \mathrm{Cl}$ & $P$ value \\
\hline \multicolumn{4}{|l|}{ Model A } \\
\hline Sex & 1.61 & 1.03 to 2.52 & 0.037 \\
\hline Age & 1.06 & 1.04 to 1.09 & $<0.001$ \\
\hline $\mathrm{GH}^{\mathrm{a}}$ & 0.62 & 0.49 to 0.77 & $<0.001$ \\
\hline \multicolumn{4}{|l|}{ Model B } \\
\hline Sex & 1.69 & 1.07 to 2.68 & 0.026 \\
\hline Age & 1.07 & 1.04 to 1.09 & $<0.001$ \\
\hline PCS & 0.97 & 0.95 to 0.99 & $<0.001$ \\
\hline MCS & 0.96 & 0.94 to 0.98 & $<0.001$ \\
\hline \multicolumn{4}{|l|}{ Model C } \\
\hline Sex & 1.74 & 1.11 to 2.74 & 0.016 \\
\hline Age & 1.06 & 1.04 to 1.09 & $<0.001$ \\
\hline APACHE II & 0.09 & 1.05 to 1.13 & $<0.001$ \\
\hline \multicolumn{4}{|l|}{ Model D } \\
\hline Sex & 1.80 & 1.13 to 2.86 & 0.013 \\
\hline Age & 1.06 & 1.04 to 1.09 & $<0.001$ \\
\hline $\mathrm{GH}^{\mathrm{a}}$ & 0.60 & 0.48 to 0.76 & $<0.001$ \\
\hline APACHE II & 1.09 & 1.06 to 1.14 & $<0.001$ \\
\hline \multicolumn{4}{|l|}{ Model E } \\
\hline Sex & 1.89 & 1.17 to 3.05 & 0.009 \\
\hline Age & 1.06 & 1.04 to 1.09 & $<0.001$ \\
\hline PCS & 0.97 & 0.95 to 0.99 & $<0.001$ \\
\hline MCS & 0.96 & 0.94 to 0.98 & 0.001 \\
\hline APACHE II & 1.09 & 1.05 to 1.13 & $<0.001$ \\
\hline
\end{tabular}

aGeneral Health $(\mathrm{GH})$ is item 1 from the SF-36: range 1 (poor) to 5 (excellent). The ranges for PCS and MCS are both 0 to 100 . Model A included the general health item of the 36-item Short-form (SF-36), age and sex. Model B included the physical component score (PCS), mental component score (MCS), age and sex. Model C included the Acute Physiology and Chronic Health Evaluation (APACHE) II score, age and sex. Model D included the general health item of the SF-36, APACHE II score, age and sex. Model E included PCS, MCS, APACHE II score, age and sex. $\mathrm{Cl}$, confidence interval; OR, odds ratio.

More recently, Welsh and coworkers [25] found that baseline patient functional status, as assessed by care providers, is correlated with mortality after ICU admission. However, that study is hampered by several drawbacks. Although the investigators also focused on patients with an expected ICU stay longer than 48 hours, they included only $9 \%$ of all ICU patients, which may indicate at least some form of selection bias. In addition, it may be questionable to correlate HRQOL scores directly with APACHE II scores without making any attempt to correct for confounding by multivariate analysis. Also, hospital deaths were not included in their analysis, which makes it difficult to understand the relation between HRQOL before ICU admission and mortality during or after critical illness.

The most recent work on this issue is that reported by RiveraFernandez and coworkers [26], who demonstrated in a multicentre study that HRQOL before ICU admission is related to ICU mortality, but that it contributes little to the discriminatory ability of the APACHE III prediction model and has little influence on ICU resource utilization, as indicated by length of stay in the ICU or therapeutic interventions [26]. However, the cohort they evaluated is not comparable with our patients, 
Figure 2

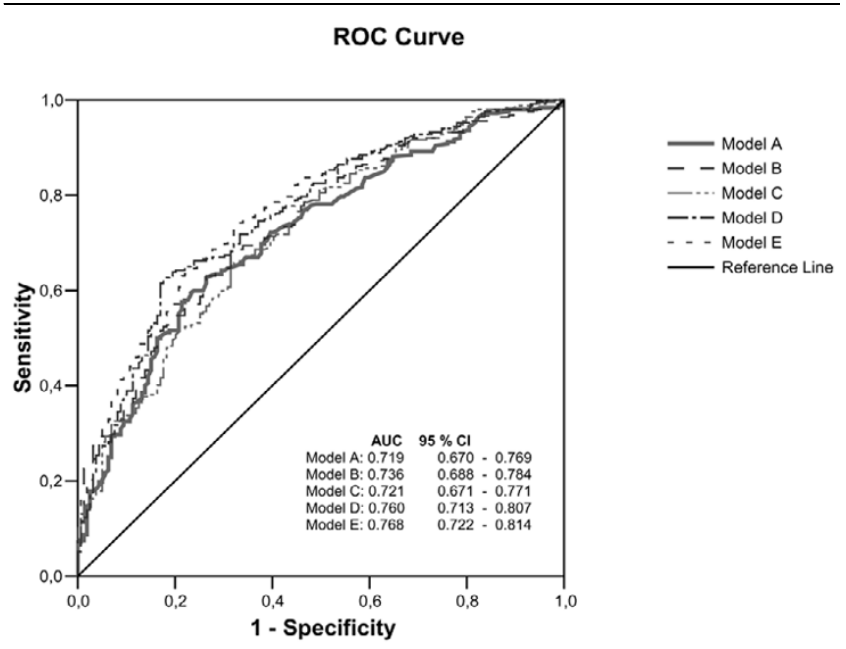

Receiver operating characteristic analysis of pre-admission HRQOL and APACHE II scores in relation to mortality. A total of 451 critically ill patients were included in the analysis. Model $A$ included the general health item of the 36-item Short-form (SF-36), age and sex. Model B included the physical component score (PCS), mental component score (MCS), age and sex. Model $\mathrm{C}$ included the Acute Physiology and Chronic Health Evaluation (APACHE) II score, age and sex. Model D included the general health item of the SF-36, APACHE II score, age and sex. Model E included PCS, MCS, APACHE II score, age and sex. $\mathrm{Cl}$, confidence interval; HRQOL, health-related quality of life; ROC, receiver operating characteristic.

because at least $25 \%$ of the patients were admitted with a cardiac diagnosis, probably because coronary care units also participated in the study. Consequently, the number of surgical patients was only $24 \%$, which is much lower than in a general ICU. In addition, the APACHE III score was used and related to a self-developed HRQOL questionnaire. Despite the differences that exist between these previous reports and ours, their findings are generally in accordance with ours and indicate that estimation of HRQOL before ICU admission deserves more attention by those caring for critically ill patients.

We conducted a long-term prospective study, which is an important strength of the data presented. Nevertheless, several limitations of our study should be mentioned. First, potential selection bias might have been present, because the HRQOL assessment could have influenced the decision to admit a patient to the ICU. However, we do not believe that this factor is important because the research nurse conducting the study did not communicate HRQOL findings to attending ICU physicians. Second, the APACHE II system was intended to be used to predict in-hospital mortality, not longterm mortality at 6 months or even later. However, repeating the analysis when omitting those patients who died after hospital discharge did not alter the results.

A third limitation of our study was the necessary use of proxies to evaluate pre-admission HRQOL instead of a retrospective assessment at ICU discharge could also have hampered results. We believe that this approach did not affect the final results, in view of the findings of previous validation studies [911]. Moreover, the use of proxies appears to be sensible, because critical illness itself could have influenced patients' recollections of their pre-admission health status. However, other groups have raised concerns about proxy estimations of HRQOL in populations with greater disease severity [27]. The same study suggested that predictions of poor ICU outcome may be exaggerated if proxies underestimate HRQOL. However, in contrast to the situation in our previous validation study, in which patients and their proxies were interviewed within 72 hours of ICU admission, these investigators interviewed patients 3 months after ICU discharge, and their proxies at study entry. This makes it entirely possible that survivors of critical illness may overestimate pre-admission HRQOL.

A fourth limitation is that we only included patients with an ICU stay longer than 48 hours, because we aimed to evaluate in particular the sickest patients surviving critical illness. Clearly, this selection makes definite conclusions regarding HRQOL as a predictor of mortality impossible. Nevertheless, the combination of the APACHE II score with HRQOL scores improved the correct prediction of survival. A final potential limitation of the study is that this was a single centre study and the results may not be generalizable to other ICU populations with different patient populations or staffing situations.

\section{Conclusion}

Pre-admission HRQOL, as estimated using a single-item question, in critically ill patients is as good at predicting survival/mortality as the APACHE II score. Initial evaluation of HRQOL can be done with the single-item question, because the SF-36 (PCS and MCS) yielded comparable results. The value in clinical practice of using the pre-admission HRQOL (PCS, MCS and general question) and the APACHE II score to provide useful predictive information in order to inform decision making appears to be limited, because of limitations in these models' abilities to predict survival/mortality in individual cases. Incorporating HRQOL into prediction models does not improve the predictive capacity of established models such as the APACHE II score. Nevertheless, it appears sensible to incorporate assessment of HRQOL into the many variables that may be considered when deciding whether a patient should be admitted to the ICU.

\section{Key messages}

- Estimate of HRQOL before ICU admission is as good at predicting survival/mortality as the APACHE II score.

- The value of HRQOL measures and the APACHE II score is limited in clinical practice for making decisions in individual cases. 


\section{Competing interests}

The authors declare that they have no competing interests.

\section{Authors' contributions}

All authors contributed substantially to the study. JGMH analyzed and interpreted the data and drafted the manuscript. PES conceived of the study, contributed to the interpretation and analysis of the data, and revised the manuscript for important intellectual content. JHR conceived of the study, contributed to its design and the interpretation of the data, and revised the manuscript for important intellectual content. HFvS conceived of the study, contributed to the analysis and interpretation of the data, and revised the manuscript for important intellectual content. AJPS contributed to the interpretation of the data, and revised the manuscript for important intellectual content. JB contributed to the design and the interpretation of the data, and revised the manuscript for important intellectual content. All authors approved the final version submitted for publication.

\section{References}

1. Knaus WA, Wagner DP, Zimmerman JE, Draper EA: Variations in mortality and length of stay in intensive care units. Ann Intern Med 1993, 118:753-761.

2. Angus D, Ishizaka A, Matthay M, Lemaire F, Macnee W, Abraham E: Critical care in AJRCCM 2004. Am J Respir Crit Care Med 2005, 171:537-544.

3. Reis Miranda D, Gyldmark M: Evaluating and Understanding of Costs in the Intensive Care Unit. In Current practice in Critical IIIness Edited by: Ryan DW. London: Chapman \& Hall; 1996:129-149.

4. Anonymous: Predicting outcome in ICU patients. 2nd European Consensus Conference in Intensive Care Medicine. Intensive Care Med 1994, 20:390-397.

5. Knaus WA, Draper EA, Wagner DP, Zimmerman JE: APACHE II: a severity of disease classification system. Crit Care Med 1985, 13:818-829.

6. Ware JE: Health Survey Manual and Interpretation Guide Waltham, MA: Medical Outcomes Trust; 1993.

7. Ware JE Jr, Kosinski M, Bayliss MS, McHorney CA, Rogers WH, Raczek A: Comparison of methods for the scoring and statistical analysis of SF-36 health profile and summary measures: summary of results from the Medical Outcomes Study. Med Care 1995, 33:AS264-AS279.

8. Aaronson NK, Muller M, Cohen PD, Essink-Bot ML, Fekkes M, Sanderman R, Sprangers MA, te Velde A, Verrips E: Translation, validation, and norming of the Dutch language version of the SF-36 Health Survey in community and chronic disease populations. J Clin Epidemiol 1998, 51:1055-1068.

9. Hofhuis J, Hautvast JL, Schrijvers AJ, Bakker J: Quality of life on admission to the intensive care: can we query the relatives? Intensive Care Med 2003, 29:974-979.

10. Capuzzo M, Grasselli C, Carrer S, Gritti G, Alvisi R: Quality of life before intensive care admission: agreement between patient and relative assessment. Intensive Care Med 2000, 26:1288-1295.

11. Rogers J, Ridley S, Chrispin P, Scotton H, Lloyd D: Reliability of the next of kins' estimates of critically ill patients' quality of life. Anaesthesia 1997, 52:1137-1143.

12. Diehr P, Patrick DL, Spertus J, Kiefe Cl, McDonell M, Fihn SD: Transforming self-rated health and the SF-36 scales to include death and improve interpretability. Med Care 2001, 39:670-680.

13. Sloan JA, Aaronson N, Cappelleri JC, Fairclough DL, Varricchio C: Assessing the clinical significance of single items relative to summated scores. Mayo Clin Proc 2002, 77:479-487.

14. Knaus WA, Wagner DP, Draper EA, Zimmerman JE, Bergner M, Bastos PG, Sirio CA, Murphy DJ, Lotring T, Damiano A, et al:: The
APACHE III prognostic system. Risk prediction of hospital mortality for critically ill hospitalized adults. Chest 1991 , 100:1619-1636.

15. Lemeshow S, Teres D, Klar J, Avrunin JS, Gehlbach SH, Rapoport $\mathrm{J}$ : Mortality Probability Models (MPM II) based on an international cohort of intensive care unit patients. JAMA 1993, 270:2478-2486.

16. Le Gall JR, Lemeshow S, Saulnier F: A new Simplified Acute Physiology Score (SAPS II) based on a European/North American multicenter study. JAMA 1993, 270:2957-2963.

17. Patel PA, Grant BJ: Application of mortality prediction systems to individual intensive care units. Intensive Care Med 1999, 25:977-982.

18. DeOreo PB: Hemodialysis patient-assessed functional health status predicts continued survival, hospitalization, and dialysis-attendance compliance. Am J Kidney Dis 1997, 30:204-212.

19. Kalantar-Zadeh K, Kopple JD, Block G, Humphreys MH: Association among SF36 quality of life measures and nutrition, hospitalization, and mortality in hemodialysis. J Am Soc Nephrol 2001, 12:2797-2806.

20. Lowrie EG, Curtin RB, LePain N, Schatell D: Medical outcomes study short form-36: a consistent and powerful predictor of morbidity and mortality in dialysis patients. Am J Kidney Dis 2003, 41:1286-1292.

21. Rumsfeld JS, MaWhinney $S$, McCarthy M Jr, Shroyer AL, VillaNueva CB, O'Brien M, Moritz TE, Henderson WG, Grover FL, Sethi GK, Hammermeister KE: Health-related quality of life as a predictor of mortality following coronary artery bypass graft surgery. Participants of the Department of Veterans Affairs Cooperative Study Group on Processes, Structures, and Outcomes of Care in Cardiac Surgery. JAMA 1999, 281:1298-1303.

22. Konstam V, Salem D, Pouleur H, Kostis J, Gorkin L, Shumaker S, Mottard I, Woods P, Konstam MA, Yusuf S: Baseline quality of life as a predictor of mortality and hospitalization in 5,025 patients with congestive heart failure. SOLVD Investigations. Studies of Left Ventricular Dysfunction Investigators. $A m ~ J$ Cardiol 1996, 78:890-895.

23. Maisey NR, Norman A, Watson M, Allen MJ, Hill ME, Cunningham $D$ : Baseline quality of life predicts survival in patients with advanced colorectal cancer. Eur J Cancer 2002, 38:1351-1357.

24. Yinnon A, Zimran A, Hershko C: Quality of life and survival following intensive medical care. OJM 1989, 71:347-357.

25. Welsh $\mathrm{CH}$, Thompson K, Long-Krug S: Evaluation of patient-perceived health status using the Medical Outcomes Survey Short-Form 36 in an intensive care unit population. Crit Care Med 1999, 27:1466-1471.

26. Rivera-Fernandez R, Sanchez-Cruz JJ, Abizanda-Campos R, Vazquez-Mata G: Quality of life before intensive care unit admission and its influence on resource utilization and mortality rate. Crit Care Med 2001, 29:1701-1709.

27. Scales DC, Tansey CM, Matte A, Herridge MS: Difference in reported pre-morbid health-related quality of life between ARDS survivors and their substitute decision makers. Intensive Care Med 2006, 32:1826-1831. 